\title{
2 Erkenntnistheoretischer und sprachtheoretischer Rahmen
}

\subsection{Diskurswelten: Sprache - Denken- Wirklichkeit}

\subsubsection{Wissen zwischen Realität und (Sprach-)Zeichen}

Wissen wird in unserer heutigen Gesellschaft eine große Bedeutung beigemessen (vgl. Felder 2013: 13; Konerding 2009: 79, Felder 2009a: 21), was nicht zuletzt in geflügelten Worten wie Wissen ist Macht deutlich zum Ausdruck kommt. Fragt man, wie Wissen bzw. Erkenntnis über die Welt entsteht, so spiegelt sich darin ein Spannungsverhältnis zwischen Innen- und Außenwelt wider. Das Wort Wissen, das etymologisch auf die ,indogermanische Präteritalform voida (= gesehen haben)“ zurückgeführt wird (Konerding 2009: 80), verdeutlicht die enge Verbindung zu Fragen der visuellen Wahrnehmung. Das Wort Erkenntnis mit dem darin enthaltenen Verb kennen ist „sprachhistorisch mit können verwandt“ (ebd.). Können verbindet man im Sprachgebrauch mit Tätigkeiten oder Praktiken, es verweist demnach auf eine vorgängige Praxis, die auf einem „[V]ertraut (gemacht worden) sein mit" (ebd.) basiert. ${ }^{46}$

Um die verschiedenen bis in die Gegenwart nebeneinander bestehenden Ansichten, wie Individuen und Welt miteinander in Beziehung treten und welche Rolle Sprache und Handeln für Erkenntnis und Wissen zukommen, einzuordnen, kann zunächst die Perspektive der Ontogenese menschlicher Erkenntnis- und Sprachfähigkeit aufschlussreich sein.

Köller beschreibt in Auseinandersetzung mit Arbeiten Jean Piagets, Jerome Bruners und Lew Wygotskis kindliche Formen „des perspektivischen Hineingleitens in die Welt“ (Köller 2004: 140). Kinder hätten eine unmittelbare „Wahrnehmung von Gegebenheiten an sich“ (ebd.: 149) und „die Einordnung der Dinge in kognitive Schemata“ würde sich „weniger nach objektiven Merkmalen der Dinge selbst“ richten, sondern eher danach, „welche subjektive Relevanz“ die Dinge für die Kinder haben und ob die Dinge in konkreten, zu bewältigenden, sinnlichen Handlungsbezügen erscheinen (ebd.: 144). Dies führe zur Bildung eigener kognitiver Muster, die sich von denen Erwachsener unterscheiden. Clark (1973: 79-83, zitiert nach Szagun ${ }^{5}$ 2013: 140) beschreibt beispielsweise Überdehnungsfehler bei Kindern im Alter von 1-2,5 Jahren (vgl. zusammenfassend Szagun ${ }^{5}$ 2013: 140f.).

46 Zur Etymologie von kennen und können und deren „gedankliche Zusammenhänge“ in Bezug auf „Wissen“ siehe Jochen Bär (2015: 3).

Ә Open Access. (C) 2021 Theresa Schnedermann, publiziert von De Gruyter. (c) BY-NC-ND Dieses Werk ist lizenziert unter einer Creative Commons Namensnennung-Nicht-kommerziell-Keine Bearbeitung 4.0 International Lizenz. https://doi.org/10.1515/9783110727838-002 
Kinder übertragen dabei ein gelerntes ,Wort ${ }^{\star}, \mathrm{d}$. h. die Verbindung eines Lautgebildes mit einer kognitiven Einheit, auf andere Gegenstände, weil sie sie semantisch ähnlich finden. Ein Kind bezeichnet beispielsweise einen vorbeifahrenden Zug mit sch, und überdehnt diese Bezeichnung, indem es sie auf alle sich bewegenden Maschinen anwendet (ebd.). Für Außenstehende ist diese Ähnlichkeitsbeziehung „kaum nachvollziehbar, aber im Rahmen der Wahrnehmungsperspektiven des Kindes durchaus verständlich“ (Köller 2004: 145). Dieses Beispiel zeigt, dass Kinder in diesem Alter noch andere Kategorisierungsstrategien zur Begriffsbildung benutzen als Erwachsene. Im weiteren Spracherwerb passen sie sich jedoch mehr und mehr den

Objektivierungsstrategien der Erwachsenen an und übernehmen deren Wahrnehmungsstrategien. Durch den schulischen Unterricht und den Erwerb der Schriftsprache wird dieser Prozess dann sehr verstärkt. ${ }^{47}$

(Köller 2004: 145)

Wenn man dieses Zitat von Köller semiotisch liest, so dokumentiert sich in der Entwicklung des Kindes ein Prozess, den Ch. S. Peirce „Semiose“ nannte. Die Vorliebe von Kindern für Frageketten unterstreicht förmlich diesen Vorgang, in dem ein Zeichen zum Interpretanten eines vorgängigen Zeichens wird und selbst abermals durch ein Zeichen interpretiert werden kann usw. (vgl. Felder 2012: 128). Dieser Prozess setzt sich bis zum Lebensende fort und ist im Prinzip unabschließbar, dadurch dass Menschen immer neue zeichengebundene Interpretationen von Wirklichkeit kennenlernen, produzieren und dadurch ihren Verstehens- und Interpretationshorizont stetig erweitern und verändern. Auf das Zeichenmodell von Peirce wird in Kap. 2.2.2 noch ausführlicher eingegangen.

Mit der ontogenetischen Entwicklung menschlicher Erkenntnis- und Kommunikationsmöglichkeiten könnte es zusammenhängen, dass Menschen aus

47 Köller bezieht sich dabei auf Lew Wygotski, für den die „Phase des begrifflichen Denkens“ darin bestehe, dass „die Kinder bei der Bildung kognitiver Muster subjektiv motivierte und bedingte Differenzierungskriterien mehr und mehr aufgeben und sich zunehmend an den Sachmerkmalen der Dinge orientieren“ (Köller 2004: 145; Wygotski ${ }^{31971: ~ 119 f f .) . ~ K o ̈ l l e r ~ w e i s t ~ j e d o c h ~}$ darauf hin, dass auch diese Begriffsbildung ,ebenfalls perspektivisch von bestimmten Wahrnehmungsinteressen geprägt ist“, die eine gewisse kollektive Akzeptanz besitzen. Für den Einfluss der Schulbildung führt Köller zudem eine Studie des russischen Psychologen Aleksandr R. Lurija aus den 30er Jahren an, der anhand zweier Versuchspersonengruppen in Usbekistan (ländliche analphabetische Versuchspersonen ohne Schulbildung und Versuchspersonen mit Schulbildung) gezeigt habe, dass diese Versuchspersonengruppen Wörter unterschiedlich kategorial zu Komplexen zusammenfassten. Bei der ersten Gruppe seien eher die „konkreten Handlungssituationen“ für Ähnlichkeitsüberlegungen und bei der zweiten Gruppe eher „Aspekte [...], die in situationsabstrakten theoretischen Betrachtungsweisen“ hervortreten, wichtig gewesen (Köller 2004: 146 mit Bezug auf Lurija 1986: 14 und 115ff.). 
ihrer alltagsweltlichen Erfahrung sowohl die Aussage unterstreichen würden, dass Wahrnehmungs- und Erkenntnisvorgänge unmittelbar ablaufen, als auch, dass Wahrnehmung und Erkenntnis von Faktoren abhängen, die sich zwischen Subjekt und Objekt schieben. Diese Faktoren werden immer dann besonders deutlich, wenn Menschen merken, dass sie sich getäuscht haben oder erkennen, dass andere Menschen eine Sache anders wahrnehmen als sie selbst.

Auf der einen Seite bewahren sich Menschen also diesen ,kindlich' unmittelbaren Zugang zur Welt zeitlebens:

Wir nehmen die Welt als gegeben an und halten ein unmittelbares Erkennen ihrer Phänomene sehr wohl für möglich. Eine (einzel)sprachliche Prägung von Erkenntnisvorgang und -resultat scheint dagegen in aller Regel nicht stattzufinden.

(Felder/Gardt 2015: 4)

Diese Annahme entspricht einer realistischen Weltauffassung, die davon ausgeht, dass es Menschen möglich ist, durch den menschlichen Wahrnehmungsapparat und in der empirischen Forschung durch geeignete technische Hilfsmittel wie das Mikroskop den Dingen der Welt aus verschiedenen Perspektiven so nahe $\mathrm{zu}$ kommen, dass man sich weitgehend objektive Vorstellungen davon bilden kann. Die Abbildung der wahrgenommenen Objekte durch Zeichen (Diagramme, (formal)sprachliche Zeichen) ist im Anschluss daran adäquat, wenn die Abbildung mit der Realität möglichst genau übereinstimmt bzw. korrespondiert (vgl. Köller 2004: 210f.). Durch die Ausbildung einer „lingua rationalis [...], für die es seit dem 17. Jahrhundert immer wieder Entwürfe und Vorschläge gab“ (Felder/ Gardt 2015: 10), sollen „drohende[n] Täuschungen“ durch die Alltagssprache (vgl. ebd. und Köller 2004: 211) verhindert werden. ${ }^{48}$ Stattdessen sollen die im Prinzip objektiven, von Interessen und Sprache unabhängigen Beobachtungen in adäquate formal- bzw. beobachtungssprachliche Zeichen übersetzt und in Definitionen kondensiert werden.

Auf der anderen Seite zeigt die ontogenetische Entwicklung kindlicher zu erwachsener Begriffsbildung jedoch anschaulich, wie Begriffssysteme sich je nach Standpunkt, Wahrnehmungsbedingungen, Lebenssituation, -raum und natürlich auch gemessen am kognitiven und sprachlichen Entwicklungsstand grundlegend ändern.

48 Im Fach Psychologie besitzt die Diskussion über eine präzise Fachsprache besondere Brisanz, da die alltagsweltlichen, mit subjektiven Wertungen verbundenen Redeweisen über innere psychische Zustände stark in das Fach hineinwirken, die fachsprachlichen Benennungen sich davon jedoch abzugrenzen versuchen. Im Umfeld der frühen behavioristisch-psychologischen Forschung gab es Bestrebungen in Anknüpfung an Überlegungen des Logischen Empirismus eine Protokollsprache für die Aufzeichnung von Verhaltensbeobachtungen $\mathrm{zu}$ entwickeln (vgl. Mandler/Kessen 1959: 40ff.; Steinig 1981: 425; Herzog 2012: 62ff.). 
Annahmen, die an diese Beobachtung anschließen und der Ansicht sind, dass Menschen nicht unverstellt auf die Welt zugreifen können, sondern unterschiedliche Faktoren konstruktiven Einfluss auf die Wahrnehmung und kognitive Verarbeitung nehmen (z. B. die neurobiologische Ausstattung des Menschen, Situationsaspekte, soziale und kulturelle Komponenten, sprachliche Komponenten), werden in der aktuellen wissenschaftlichen Diskussion als konstruktivistisch bezeichnet (vgl. Felder/Gardt 2015: 4). Die Grundannahme dieser Ansätze lässt sich auf Kants sogenannte „kopernikanische Wende“ zurückbeziehen, die Immanuel Kant in der Vorrede zur „Kritik der reinen Vernunft“ damit beschreibt, dass sich die Anschauung nicht „nach der Beschaffenheit der Gegenstände“, sondern „der Gegenstand (als Objekt der Sinne) nach der Beschaffenheit unseres Anschauungsvermögens“ richte (Kant [21787] 1989: B XVI-XVII; vgl. Felder/Gardt 2015: 9).

In den Geistes- und Sozialwissenschaften gilt dieses Modell als das aktuell vorherrschende Paradigma (vgl. Felder/Gardt 2015: 4), aber auch in Lehrbüchern der allgemeinen Psychologie, die sich selbst zu großen Teilen auf das Paradigma empirischer Wissenschaft rückbezieht, wird zur Beschreibung des Phänomens WAHRNEHMUNG von „einem aktiven und konstruktiven Akt“ ausgegangen und „um den Bezug zwischen der Wahrnehmung und der Handlung“ zu beschreiben, werden „Prozesse der Aufmerksamkeit und der Aufmerksamkeitskontrolle“ eingehend behandelt (Hagendorf et al. 2011a: VI). ${ }^{49}$

Von der jeweiligen Annahme, ob man ein unmittelbares Erkennen der Welt für möglich hält oder nicht, hängt u.a. ab, welche Antworten man auf „das Problem der Gültigkeit von Wahrnehmungs- und Wissensinhalten“ (Köller 2004: 209) und ihrer Repräsentation geben kann. Realistische Weltauffassungen, die eine direkte referentielle Bezugnahme der Zeichen auf die transsemiotische Welt für möglich halten, tendieren dazu, eine

Wirklichkeit vorauszusetzen, die unabhängig von Zeichenprozessen, vielleicht sogar unab-
hängig von Erkenntnisprozessen als eine gegliederte und strukturierte Realität gegeben ist.
Zeichen können nur direkt auf Gegenstände der Welt Bezug nehmen, wenn diese (unabhän-
gig von Zeichenprozessen) als unterscheidbar vorliegen.
(Jäger 2010a: 40)

49 Einen Überblick zu verschiedenen realistischen und konstruktivistischen Strömungen und diesen erkenntnistheoretischen Diskurs prägenden Publikationen findet man bei L. Jäger 2010a: 32ff. Jäger konstatiert dort, dass spätestens seit Karl Popper die „Maxime, dass (wissenschaftliche) Beobachtung immer Beobachtung im Lichte von Theorien sei“ auch in die „Forschungslogik der Naturwissenschaften“ eingegangen sei (Jäger 2010a: 36 mit Bezug auf Popper 61976: 31). Die wissenschaftstheoretischen Publikationen von Ludwik Fleck binden die Erkenntnismöglichkeiten in der Medizin ebenfalls an „Denkstile“ (siehe dazu auch Kap. 3.2 dieser Arbeit). 
Die repräsentierenden Zeichen werden überwiegend danach beurteilt, wie adäquat sie die Wirklichkeit stellvertretend abbilden.

Dieses Modell berücksichtigt nach Jäger zu wenig „die zweite Dimension des Verhältnisses von Zeichen und Wirklichkeit: nämlich die Dimension, in der sich Zeichen auf andere Zeichen beziehen“ (Jäger 2010a: 39). Jäger vertritt die These, dass

Zeichen-Zeichen-Beziehungen als wichtig, vielleicht sogar als unverzichtbar dafür angesehen werden, dass wir überhaupt in das Feld des Wirklichen Unterscheidungen eintragen können. Die Unterscheidung von Dingen und Sachverhalten würde in diesem Sinne als ein Ergebnis der inferentiellen Zeichenbeziehungen angesehen.

(Jäger 2010a: 40)

Aktuelle psychologische Modelle zur Funktionsweise der (visuellen) Wahrnehmung unterstützen diese These dadurch, dass sie die Wahrnehmung als mehrstufigen Prozess beschreiben (sensorische Prozesse, perzeptuelle Organisation, Identifikation/Wiedererkennen), in dem verschiedene Vorgänge zusammenwirken müssen, damit ein Wahrnehmungsobjekt entsteht (vgl. Gerrig/Zimbardo ${ }^{20} 2015$ : 112ff.). So muss eine mentale Repräsentation eines Gegenstandes, beispielsweise verschiedene Linien, die ein Viereck bilden, erst mit Umgebungsreizen und einem bekannten Konzept verbunden werden, um als >Fenster und nicht als >Bild an der Wand identifiziert werden zu können. Nimmt man mit dem Sprachzeichen Fenster und dem damit verbundenen Konzept auf den Sachverhalt Fenster Bezug, so ist dies bezogen auf die semiotische These Jägers möglich, weil

[D]ie semantischen Gehalte der Zeichen, mit denen Sprecher einen solchen Bezug herstellen, in vergangenen inferentiellen Bezugnahmen von Zeichen auf Zeichen möglich gemacht worden sind. [...] Unsere kulturellen Semantiken verdanken sich nicht einer ontologischen Ordnung der ,Welt selbst'. Ihre Funktion kann sich nicht darin erschöpfen, Weisen bereitzustellen, in denen eine transsemiotische (,ontologische') Welt dargestellt, abgebildet, gespiegelt zu werden vermag. Wir verfügen - so Rorty - über keinen ,transzendentalen Standpunkt außerhalb unserer gegenwärtigen Darstellungssysteme, von dem aus wir die Relation zwischen diesen Darstellungen und ihrem Gegenstand untersuchen könn[t]en“50, über keinen exzentrischen archimedischen Punkt also, der es uns erlaubte, die Adäquatheit unserer Bezugnahmen auf die Welt unabhängig von medialen Darstellungssystemen zu beurteilen. Wir müssen uns vielmehr auf Semantiken stützen, für die das Interpretations-Prinzip konstitutiv ist.

(Jäger 2010a: 45f.)

50 Jäger zitiert hier in der Fußnote Rorty (1987: 321). Im Literaturverzeichnis dieser Arbeit aufgenommen unter Rorty 1981: 321. 
Im pansemiotischen Modell von Peirce, auf das in Kap. 2.2.2 noch weiter eingegangen wird, liest sich dies folgendermaßen:

Aus der Tatsache, dass jeder Gedanke ein Zeichen ist, folgt dass der Gedanke einen weiteren Gedanken adressieren muss, weil darin das Wesen des Zeichens besteht. Jeder Gedanke muss durch einen anderen Gedanken interpretiert worden sein. ${ }^{51}$

(Peirce 1984: 173, Übersetzung von Jäger 2010a: 44)

In diesen Ansätzen, die (Sprach-)zeichen eine erkenntniskonstitutive Rolle zusprechen, wird die Repräsentation von Wissensinhalten durch Zeichen nicht als reine Stellvertretung, sondern als Vermittlung oder auch Übersetzung bzw. „Transkription“ (vgl. Jäger 2012a) betrachtet: aktuelle Objektivierungsbemühungen manifestieren sich in Zeichen, diese bedienen sich dabei vorgängiger kollektiv bewährter, zeichengebundener Objektivierungsmöglichkeiten. Sie setzen das Repräsentierte des Weiteren in Bezug zu denjenigen, die sich und anderen etwas repräsentieren (vgl. Köller 2004: 219).

Nicht mehr (ausschließlich, T.S.) die „Konfrontation, das Gegenüberstellen von Personen und Sachverhalten“ bestimmt das Szenario des Erkennens, sondern die „Kommunikation, das Gespräch zwischen Personen“, die sich über das verständigen, was erkennend verstanden wird [aufgelöste Fußnote: Rorty 1987: 191].

(Jäger 2010a: 37)

Wissen basiert in diesen Ansätzen auf „kollektive[n] Meinungen, die als unstrittig geteilt werden und über die ein relativer Konsens besteht“ (Konerding 2009: 81). Damit sie kollektiv geteilt und als Wissen, auf das man sich bewusst beziehen kann, etabliert und gesichert werden können, ist ein kommunikativer Austausch über dieselben notwendig.

Diese soeben dargestellten Theorien des Wirklichkeitsbezugs und die damit zusammenhängenden Wahrheits- und Wissenskonzepte bestehen nebeneinander fort und müssen sich nicht gänzlich ausschließen. Denn man kann (Sprach-) zeichen und anderen, z. B. sozial-kulturellen Faktoren eine erkenntniskonstitutive Rolle zusprechen, ohne „zu behaupten, dass es eine Wirklichkeit jenseits der Zeichen“ oder ihres Konstruiertseins nicht gebe (Jäger 2010a: 38; vgl. Felder/Gardt 2015: 14). Des Weiteren sind die verschiedenen Theorien des Wirklichkeitszugriffs für die Diskursanalyse im Praxisteil dieser Arbeit von großer Bedeutung, da sich ihre Spuren in der definitorischen Konstituierung des Phänomens BuRnouT und in sprachlichen Mitteln des Geltungsanspruchs niederschlagen.

51 Im Original liest sich diese Passage folgendermaßen: „From this proposition that every thought is a sign it follows that every thought must address itself to some other, must determine some other, since that is the essence of a sign.“ (CP 5.253; Peirce 1984: 173). 
Auch wenn man die „Möglichkeit sprachfreien Denkens“ (Funke ${ }^{4} 2011:$ 124) anerkennt, so gehen doch viele Theorien zum Zusammenhang zwischen Sprache und Denken von „enge[n] genetische[n] und funktionale[n] Beziehungen“ bzw. einer „Leistungssymbiose“ zwischen diesen menschlichen Fähigkeiten aus (ebd.). In experimentellen psychologischen Studien wurde Sprache als das Denken befördernde und hemmende Kraft nachgewiesen (Funke ${ }^{4} 2011$ : 127/128). Sprache - auch in nicht artikulierter Form, sondern als inneres Selbstgespräch - steuere beispielsweise Problemlösungsprozesse. Sprachliche Zeichen werden dabei als „konstitutive Mittel angesehen, um Vorstellungsinhalte zu objektivieren und zu vermitteln“ (Köller 2004: 3). Sprache könne aber auch hemmend auf das Problemlösen wirken, wenn „durch eine sprachliche Etikettierung bestimmte Lösungsmöglichkeiten nicht in Erwägung gezogen werden“ (Funke ${ }^{4} 2011: 128$ mit Bezug auf Duncker 1935/31974). Im letzten Beispiel zeigt sich die perspektivierende Kraft sprachlicher Zeichen. Diese wird bei jeder Wahl eines bestimmten Vokabulars und bestimmter grammatischer Formmuster zur Darstellung eines Sachverhalts, die Menschen spontan-unbewusst oder überlegt-bewusst treffen, wirksam (vgl. Köller 2004: 22). Sie lenkt die Aufmerksamkeit bzw. kognitive Einstellung zunächst einmal auf einen bestimmten Aspekt des wahrgenommenen Objekts und bewirkt, dass andere Perspektiven in diesem Moment ausgeblendet werden (vgl. Felder 2008: 271; Köller 2004: 3). Bei Texten, die man rezipiert, um sich über ein Thema zu informieren, wodurch man jenseits eigener Primärerfahrung sich Wissen durch Beschreibung aneignet (vgl. Warnke 2009: 122ff.; Felder/Gardt 2015: 15), tritt die perspektivierende Kraft sprachlicher Zeichen noch deutlicher zutage.

Im folgenden Kapitel wird anhand von Beispielen noch ausführlicher erläutert, worin sich die kommunikative und kognitive Perspektivität sprachlicher Zeichen zeigt.

\subsubsection{Kognitive und kommunikative Perspektivität sprachlicher Zeichen}

Wilhelm Köller, auf den die im Weiteren zu erläuternde Unterscheidung der „kommunikativen“ und „kognitiven Perspektivität“ sprachlicher Zeichen zurückgeht, sieht Sprache als „operativen Faktor in umfassenderen Ordnungssystemen“ (Köller 2004: 25). Er sieht in der verbundenen Betrachtung von Sprache und Perspektivität die Chance, die verschiedenen Dimensionen menschlicher Tätigkeiten bei der Repräsentation von Welt zu erfassen:

Die Sprache erscheint so gesehen als ein kognitives Werkzeug, in deren Formen sich die Ergebnisse vorangegangener Perspektivierungs- und Objektivierungsanstrengungen für eine künftige Nutzung niedergeschlagen haben. Das bedeutet, dass der Perspektivitäts- 
begriff gerade in seiner Korrelation mit dem Sprachbegriff letztendlich als ein anthropologischer Grundbegriff zu verstehen ist, weil mit ihm das Zusammenspiel biologischer, kultureller und situativer Determinanten bei der subjektiven und intersubjektiven Repräsentation von Welt erfasst wird.

(Köller 2004: 25)

Sprache wird als ein „geformter Mittler“ verstanden (Köller 2004: 310), der aktuelle mit vorangegangenen Objektivierungsanstrengungen und die verschiedenen Dimensionen, die zu einer Perspektivierung der Erfahrungs- und Vorstellungswelt führen können, miteinander verbindet. Dabei stehen die „Ergebnisse alter Perspektivierungsanstrengungen“ in einem „konstitutiven Spannungsverhältnis“ zum „Aufbau neuer Vorstellungsgestalten“. Eine „faktische Grenzlinie“ zwischen diesen beiden, kann nach Köller nicht gezogen werden (Köller 2004: 23). Die Unterscheidung basiert vielmehr auf methodischen Überlegungen, um die Wechselbeziehungen zwischen „traditionell gefestigten Sprachformen“ und ihrem ,aktuellen Gebrauch“ im Hinblick auf ihr jeweiliges Perspektivierungspotenzial beschreibbar machen zu können (vgl. Köller 2004: 309/310). Köller definiert diese beiden Kategorien wie folgt:

Von der kommunikativen Perspektivität können wir immer dann sprechen, wenn wir uns auf der Analyseebene der Sprachverwendung danach fragen, in welcher Wahrnehmungsperspektive konkrete Vorstellungsinhalte für einen Adressaten objektiviert werden. Wir interessieren uns dann für das konkrete Produkt eines sprachlichen Objektivierungs- und Sinnbildungsvorgang. Wir wollen wissen, hinsichtlich welcher Aspekte ein Sachverhalt objektiviert wird und in welcher Konstellation einzelne Elemente thematisiert werden.

(Köller 2004: 21)

Von der kognitiven Perspektivität sprachlicher Formen können wir dagegen immer dann sprechen, wenn sich unser Analyseinteresse nicht gegenstandsthematisch auf die Gestaltung konkreter Sachvorstellungen richtet, sondern reflexionsthematisch auf die konventionalisierte immanente Perspektivität der sprachlichen Muster, mit denen wir konkrete Vorstellungen objektiveren. Die Frage nach der kognitiven Perspektivität sprachlicher Formen zielt also ab auf die Struktur des kollektiven Wissens, das sich in sprachlichen Mustern verfestigt hat und das die kommunikativen Perspektivierungsmöglichkeiten dieser Muster vordeterminiert.

(Köller 2004: 22)

\section{Köller erklärt am Beispiel der Metaphern den Übergang von der kommunikativen} zur kognitiven Perspektivität:

Eine spontan neu geprägte Metapher will uns einen Sachverhalt in einer ganz bestimmten Perspektive hinsichtlich ganz bestimmter Aspekte vor Augen führen. Wenn diese Metapher zu einem konventionalisierten Sprachmuster wird, dann verändert sich die neuartige Sichtweise zu einer konventionellen. [...] Der Übergang der kommunikativen Perspektivität zur kognitiven Perspektivität einer sprachlichen Form ist eine Erscheinungsweise der kulturellen Traditionsbildung.

(Köller 2004: 22) 
In welchem Maße sich eine Sprachgemeinschaft sowohl mit „festen“ als auch mit „ad-hoc-Metaphern“ ihre „alltägliche Wirklichkeit auf ganz spezifische Weise erarbeitet und erhält“ (Liebert 1992: 1; 9), wurde in den 1970er Jahren im Rahmen der US-amerikanischen Metaphern-Diskussion eindrucksvoll verdeutlicht (vgl. Liebert 1992: 10). Der Sprachgebrauch der Wissenschaft und zwar auch derjenige der ,objektiven' Fächer ist nachweislich ebenfalls metaphorisch geprägt (Liebert 2005). Dieses Wechselspiel zwischen Konventionalisierung und aktuellem Gebrauch wird besonders bei der „innerwissenschaftliche[n] Übertragung von Deutungsmustern“ deutlich (Steinig 1981: 433). Steinig erläutert diesen Zusammenhang für psychologische Begriffe, beispielsweise für „die metaphorisierend aus der Sprache der Mechanik entlehnte Vorstellung vom Leistungsdruck“, wobei der psychologische Terminus Leistung selbst von der physikalischen Lesart geprägt ist. Dies wird im Eintrag zum Lemma Leistung des in der 17. Auflage erscheinenden „Lexikons der Psychologie“ deutlich, das an erster Stelle die Formel „physisch die in der Zeiteinheit verrichtete Arbeit $(P=W / t$, bisher: $L=A / t$ )“ zitiert. $^{52}$ Des Weiteren gibt Steinig Beispiele, die auf konzeptionelle Metaphern im Zusammenhang mit der Referenz auf den Gegenstand PsychE hindeuten. Einerseits erscheint sie als `steuernde Instanzı, die innere Zustände, z. B. Einstellungen ohne Probleme souverän ändern kann und andererseits als sunkontrollierbare Kraftı, deren Gefühle die Person überkommen oder die sie nicht fassen kann, die also ein „Eigenleben in einer räumlich gedeuteten Psyche führen“ (vgl. Steinig 1981: 435/436). Im Burnout-Diskurs findet man ähnliche Beispiele, wenn Personen auf der einen Seite ein Haushalten mit den eigenen Kräften ${ }^{53}$ empfohlen wird und auf der anderen Seite getitelt wird Ausgebrannt - Warum es kein Versagen ist, wenn Sie an Ihre Grenzen stoßen ${ }^{54}$. Ausgebrannt zu sein wird über das Wort Versagen mit Bedeutungen wie 'plötzlich aufhören zu funktionieren', 'Kontrollverlust', aber auch mit 'an etwas scheitern' in Verbindung gebracht. Dabei kommt neben der aktuell gewählten kommunikativen Perspektivierung auch die kognitiv-immanente Perspektivität zum Tragen, die sprachliche Mittel der Negation den Sprechenden bereitstellen. Negationszeichen haben sich nach Köller als „Objektivierungsstrategie[n] durch Abgrenzung“ herausgebildet (Köller 2004: 542). Sie haben ihren Ursprung in Abwehrgesten (vgl. Köller 2004: 547) und

52 Siehe Leistung in „Dorsch - Lexikon der Psychologie“ (= Wirtz/Strohmer 2019) unter https:// dorsch.hogrefe.com/stichwort/leistung (zuletzt eingesehen am 2.11.2019).

53 So in einem Fortbildungsprogramm für Mitarbeiter/innen einer Einrichtung für Behindertenhilfe im Kursangebot 47 zur Burnoutprävention: http://www.sankt-josefshaus.de/pdf/PA/ Fortbildungsprogramm_2010.pdf (zuletzt eingesehen am 10.11.2019).

54 So auf dem Titelblatt der „Psychologie Heute“, Heft 1/2016: AUSGEBRANNT - Warum es kein Versagen ist, wenn Sie an Ihre Grenzen stoßen“ (im QV unter 8.1.7). 
„[i]nformationstheoretisch sind Negationen immer in einen bestimmten Erwartungsrahmen eingebunden“ (ebd. 548). Dass AusGEBRANNT-SEIN im Sinne von 'Versagen' verstanden wird, wird demnach ,als erwartbar oder denkbar“ präsentiert (ebd.: 551), „aber faktisch“ als nicht gültig zurückgewiesen. Die Negation ist eine Praktik, die erstens die Polyphonie des Diskurses orchestriert, indem sie eine Stimme des Diskurses als Kontrapunkt zu einer anderen darstellt, zweitens durch die Bildung von Oppositionen abgrenzende, ordnende und damit auch definitorische Wirkung entfaltet (vgl. in der Zusammenfassung die Kap. 6.2.2.4 und 6.2.3.3) und drittens die Geltung der Grundinformation qualifiziert (vgl. Köller 2004: 544; Köller 1995: 39).

In der im Praxisteil dieser Arbeit folgenden Analyse zu Praktiken des Definierens am Beispiel des Burnout-Diskurses wird es folglich darum gehen, herauszuarbeiten, „in welcher Wahrnehmungsperspektive“ das Phänomen BuRNOUT im Hinblick auf verschiedene Adressaten und Kommunikationssituationen wiederholt objektiviert wird und welche konventionalisierten lexikalischen und grammatischen Muster und Sprachgebrauchspraktiken (im Prozess des Definierens) Verwendung finden und sich dadurch perspektivierend auf dessen Konstituierung im Diskurs auswirken (vgl. Kap. 6.2).

In Kapitel 2.1.1 wurden die verschiedenen Ansichten, wie Individuen und Welt miteinander in Beziehung treten können, und welche Rolle Sprache und Handeln für Erkenntnis und Wissen zukommen, ausgelotet. In den nächsten Kapiteln wird es dezidierter um die Frage gehen, wie in Diskursen Wissen entsteht. Dazu werden im folgenden Kapitel zunächst Wissensmodelle, die in linguistischen Diskursanalysen Anwendung gefunden haben, rekapituliert und diskutiert (Kap. 2.2.1). Daraufhin wird das Zeichenmodell von Charles S. Peirce vorgestellt (Kap. 2.2.2), um auf dieser Basis das semiotisch-hermeneutische Vorgehen dieser Arbeit zu entfalten (Kap. 2.3). Des Weiteren wird das Zeichenmodell von Peirce dazu dienen, die intrikate Verbindung zwischen natürlichen Zeichen (Symptomen) und darauf referierenden (non-)verbalen Zeichen beschreibungssprachlich greifbar machen zu können. Dieser Aspekt wird ausführlich in Kap. 3.1 zur medizinischen Semiotik beleuchtet.

\subsection{Zeichenvermittelte, diskursive Konstituierung von Bedeutung und Wissen}

\subsubsection{Wissensbegriffe und linguistische Diskursanalyse}

„Außerhalb der Primärerfahrung begegnet uns die Welt als eine vertextete“ (Felder 2012: 116). Felder weist mit diesem Zitat im Rahmen methodischer Überle- 
gungen für die linguistische Mediendiskursanalyse darauf hin, dass Texte unser Wissen über Sachverhalte entscheidend prägen können, ohne dass wir diese Dinge am eigenen Leib durchlebt haben müssen. Des Weiteren wird in dieser Aussage auf zwei Typen des Erwerbs von Wissen (Primärerfahrung und vertextete Weltbegegnung) aufmerksam gemacht, die Warnke mit Bezug auf Russells Unterscheidung (Russell 1911) von „knowledge by description“ und „knowledge by acquaintance“ folgendermaßen beschreibt:

Knowledge by acquaintance (Wissen aus Erfahrung) beruht auf einer unmittelbaren kausalen Interaktion zwischen Wissenssubjekt (jemand, der etwas weiß) und Wissensobjekt (das, worüber etwas gewusst wird). Als Wissensobjekte kommen beim knowledge by acquaintance stets nur sensorische Daten in Betracht, also das, was ein Subjekt sinnlich als objektiv erfährt. So ist der durch eine Person unmittelbar erfahrene Schmerz ein subjektives Wissen aus Erfahrung. Der Satz „Mein Arm schmerzt.“ ist also, wenn er durch knowledge by acquaintance verifiziert ist. Erfährt man Wissen nicht unmittelbar, sondern teilt man es ohne Erfahrung, so spricht man von knowledge by description (Wissen durch Beschreibung). Das alltagsweltliche Wissen um den Klimawandel ist für Wissenssubjekte in der Regel nicht das Ergebnis einer unmittelbaren kausalen Interaktion mit der globalen Erwärmung selbst, sondern was man weiß, weiß man aufgrund von Beschreibungen.

(Warnke 2009: 122)

In Texten über medizinisch-psychologische Themen rezipieren wir sprachlich vermitteltes Wissen über Risikofaktoren und krankhafte Symptome, ohne dieses Wissen zwingend selbst durch unmittelbare Erfahrung erworben haben zu müssen. Dieser Umstand führt zu der Frage, wie Wissen durch Beschreibung in einem Diskurs entsteht und inwiefern zuvor erworbenes Wissen durch Beschreibung unser Erleben von Schmerzen oder Risiken im Bereich von Gesundheit und Krankheit und damit auch unser Wissen aus Erfahrung beeinflussen kann.

Warnke spricht im zitierten Abschnitt und in den folgenden Passagen seines Beitrags davon, dass das knowledge by acquaintance „Wissen aus unmittelbarer sinnlicher Erfahrung“ sei und damit unberührt bleibe von diskursiven Prozessen der „Herstellung von Faktizität“, argumentativer Aushandlung und Streuung von Geltungsansprüchen. Letztere seien „Kennzeichen diskursiven Wissens“ (Warnke 2009: 123). Diese unverbundene Gegenüberstellung der Wissensarten wird hier vor dem Hintergrund der in Kap. 2.1.1 diskutierten Möglichkeiten des Weltzugriffs und aktueller psychologischer Erkenntnisse zu Prozessen der Wahrnehmung bzw. Erfahrung, die den „konstruktive[n] Charakter“ und die Komplexität von Wahrnehmungsprozessen unterstreichen (vgl. Hagendorf/Krummenacher/Müller et al. 2011b) ${ }^{55}$, in Frage gestellt, wodurch die Wahrnehmung bzw. Erfahrung dann

55 Hagendorf/Krummenacher/Müller et al. (2011b: 5) schreiben hierzu: „Die Wahrnehmungsprozesse extrahieren aus dem sich kontinuierlich verändernden, oft chaotischen Input von externen Energiequellen Bedeutungen und strukturieren sie zu stabilen, geordneten Perzepten bzw. Wahr- 
nicht mehr gänzlich 'unmittelbar' ist. Das Beispiel des Schmerzes, das Warnke anführt, spricht einerseits für seine These. Denn unsere Wahrnehmung „erfolgt in der Regel mühelos ohne erlebbare Anstrengung und ist unmittelbar“ (Hagendorf/Krummenacher/Müller 2011b: 4), und jede Person hat einen unmittelbaren Zugang zum eigenen Fühlen und Erleben (vgl. auch Winterscheid 2015: 196). Symptome, d.h. natürliche Zeichen, gelten in der (medizinischen) Semiotik im Rückgriff auf Peirce zudem als „Subtyp der Indices“ (Peirce, C.P. 8.119, vgl. dazu auch Kap. 2.2.2 und 3.1): Das Gefühl des Schmerzes steht in direkter Verbindung zum Körper und zu möglichen physischen Auslösern.

Studiendesigns, die aus einer kulturwissenschaftlichen Perspektive Schmerzen untersuchen, gehen jedoch davon aus, dass die „Reaktion auf einen physiologisch an sich gleichartigen Stimulus [...] psychologisch und soziokulturell spezifisch geformt“ ist (Lalouschek 2010: 19). Das Phänomen ScHMERz besitze zwei Gesichter:

Kleinman et al. (1992) formulieren diese Zweigesichtigkeit von Schmerz folgendermaßen: „Pain is a ubiquitous feature of human experience. (...) At the same time, the cultural elaboration of pain involves categories, idioms, and modes of experience that are greatly diverse [...].“

(Lalouschek 2010: 19.)

Wie Menschen Schmerzen wahrnehmen und mit ihnen umgehen sei auch ,,von ihrem sozio-kulturellen Hintergrund abhängig wie die Art und Weise, wie Menschen ihren Schmerz präsentieren und kommunizieren, sei es im professionellen Bereich oder im Alltag“ (ebd.: 22). ${ }^{56}$

Schmerzreize sind demnach je nach analytischer Perspektive:

- $\quad$ subjektiv unmittelbar gegebene „Daten“ oder

- intersubjektiv replizierbare „Daten“577 (durch aktuell valide medizinische Untersuchungsmethoden und mithilfe anerkannter (technischer) Geräte)

nehmungen. Ohne diesen Prozess würden wir einen Freund nicht erkennen, einen Sonnenuntergang nicht genießen können, am aufkommenden Geruch nicht erkennen können, dass das Essen auf dem Herd angebrannt ist, den Wurf eines Balls beim Basketball nicht planen können oder die Qualität eines Kleidungsstoffs nicht beurteilen können“ (Hagendorf/Krummenacher/Müller et al. 2011b: 5). „Da sich die Wahrnehmung in einem evolutionären Prozess herausgebildet hat, stecken in den Verarbeitungsprinzipien Eigenschaften im Sinne von Regularitäten der Umwelten, in denen sich dieses System bewähren musste und bewährt hat. Diese Regelhaftigkeiten der Umwelt werden als Vorannahmen im Wahrnehmungsprozess automatisch wirksam, um beispielsweise die Vieldeutigkeit der Interpretation von Reizgegebenheiten einzuschränken“ (ebd.: 6).

56 Weitere medizinische, kulturwissenschaftliche, semiotische und linguistische Perspektiven auf das Phänomen Schmerz sind im Sammelband „Sprechen über Schmerzen“ zusammengeführt (Menz/Lalouschek/Sator 2010).

57 Vergleiche die Unterscheidung in „Daten“ und „Fakten“ von Ekkehard Felder (2013: 14), die „Wissen in den epistemologisch schwierigen Zusammenhang von (Vor)Gegebenem (Daten) und 
- $\quad$ subjektiv oder intersubjektiv gedeutete „Signale“ (vgl. dazu Sebeok 1984: 42) bzw. sich körperlich materialisierende „Spuren“ (Müller 2015: 53f.), die in ein Gesetz oder eine konventionelle Deutung eingeordnet und vor dem Hintergrund von alltagsweltlichen oder nosologischen Wissensbeständen bzw. Praktiken als symptomatische „Fakten“ (vgl. Fußnote 57) eines bestimmten Krankheitsbilds/einer Verletzung gefasst werden können.

Ein Schmerz im Arm verlangt nach einer Erklärung. Ist diese nicht offensichtlich, wie z.B. durch eine Schnittverletzung, dann wird der Schmerz durch beschriebenes gelerntes Wissen und ein Hineinfühlen in sich selbst möglicherweise als ziehend und daraufhin als Symbol eines Herzinfarktes gedeutet. ${ }^{58}$ Diese Art des Hineinfühlens zum Zwecke der Deutung zeigt das „knowledge by acquaintance“ und „knowledge by description“ eng verbunden sein können. Für das Wort acquaintance findet man auf der Seite von „Lexico.com“, einem online frei zugänglichen Wörterbuch der Oxford University Press, u.a. die Synonyme familiarity, conversance, proficiency, know-how etc. ${ }^{59}$ Die Bedeutungen des 'Vertrautseins', der 'Kenntnis' bzw. des 'Könnens' als einer eigenen Wissensart neben dem Wissen aus Beschreibung ähnelt der Unterscheidung „zwischen prozeduralem und deklarativem Wissen“, die Konerding als „fundamentale Dichotomie“ seiner Konzipierung linguistischer Analyse von Wissen bzw. Wissenstransfer zugrunde legt (Konerding 2009: 83ff.). Prozedurales Wissen wird ähnlich wie das „knowledge by acquaintance“ als implizit verfügbar beschrieben. Prozedurales Wissen steht für

die jeweiligen Fähigkeiten von Individuen zweckorientiert erfolgreich zu handeln, eine spezielle Aufgabe zu meistern oder ein Problem eines bestimmten Typs erfolgreich zu lösen. Prozedurales Wissen kann partiell und selektiv in Form von sprachlichen Handlungsanweisungen ,repräsentiert' werden [...].

(Konerding 2009: 83)

Gemachtem (Fakten)“ stellt mit etymologischem Bezug auf die lateinischen Verben dare ('geben') und facere ('machen'). Wissen besteht nach dieser Dichotomie ,aus intersubjektiv unstrittig Gegebenem - also Daten als nach allgemein akzeptierten Kriterien gewonnenen, oft gemessenen Größen - [...] sowie aus Gedeutetem - also aus beobachteten Ereignissen sowie anschließend abstrahierten und damit hergestellten Tatsachen als Fakten mit breitem Gültigkeitsanspruch“ (Felder 2013: 14).

58 In Kapitel 3.1 wird die Frage, inwiefern Symptome als Indizes oder Symbole betrachtet werden können, noch einmal aufgegriffen werden.

59 Die Synonyme werden in drei Lesarten aufgeteilt: 1.) als 'Bekannter', 'Gefährte', 'Kollege' (,,contact, associate, [...] colleague“); 2. als 'Verbundenheit', 'Beziehung', 'Kameradschaft' (,association, relationship, [...] companionship“) und; und 3. als 'Vertrautsein', ‘Kenntnis’, 'Können', 'Geschick' (,familiarity, conversance, [...] knowledge of, experience of, [...] proficiency in, skill in, expertise in, insight into; informal know-how“) unter: https://www.lexico.com/en/synonym/ acquaintance (zuletzt eingesehen am 3.11.2019). 
Konerding bezeichnet „Alltagswissen“ als „zum größten Teil prozedurales Wissen“:

Alltagswissen ist eingebettet in in weiten Teilen nicht reflektierte kollektive Lebenspraxen, d. h. in konkrete Lebenssituationen und zugehörige problemlösungsbestimmte Verfahrensweisen, Verhaltensformen, -routinen und -traditionen.

(Konerding 2009: 85)

Deklaratives Wissen hingegen ist explizit und ,durch ein symbolisch vermitteltes Repräsentationsformat bestimmt“. Es entsteht aus „,symbolische[n] Modellierungen von prozeduralem Wissen, von praktischem Tun, insbesondere in dessen Regelhaftigkeiten“ und die beiden Wissensarten stehen in einem „intrikate[n] Verhältnis“ zueinander (Konerding 2009: 84; 86). Vor dem Hintergrund dieser dem Begriff Wissen zugeschriebenen Attribute stellt sich die Frage, ob eine unmittelbare Sinneswahrnehmung - ohne, dass sie (gedanklich) bezeichnet wird oder zu praktischen Konsequenzen oder mit anderen Erfahrungen verknüpften Deutungen führt - schon als Wissen bezeichnet werden kann. Entscheidend für den Begriff von Wissen, der auch dieser Arbeit zugrunde liegen soll, ist das Moment der (kollektiven) pragmatischen Orientierung ${ }^{60}$ bzw. die Entstehung „sachadäquater Handlungsformen“ und „Darstellungsformen“ (Köller 2004: 251). An das obige Beispiel anknüpfend hieße das zu wissen, wie man oder der Einzelne in der Regel mit einem schmerzenden Arm erfolgreich umgehen kann und dieses Wissen „durch sprachliche Hinweise und Erklärungen zu Funktionsweisen und zu beachtenden Zusammenhängen“ (Konerding 2009: 84) über gemeinsame Zeichen zu objektivieren, auszutauschen und zu stabilisieren. In ähnlicher Weise argumentiert Warnke an einer anderen Stelle seines Beitrags (vgl. Warnke 2009: 114) und zusammen mit Spitzmüller im Rahmen ihrer diskurslinguistischen Einführung, wenn sie Wissen als ein „sozial verhandeltes Gut der Vergesellschaftung “ beschreiben (Spitzmüller/Warnke 2011: 41). Der Begriff >Vergesellschaftung، stammt von Max Weber und meint in diesem Zusammenhang, dass Wissen das Produkt sozialen Handelns ist, das ,auf rational (wert- oder zweckrational) motiviertem Interessenausgleich oder auf ebenso motivierter Interessenverbindung beruht“ (Weber (1922) § 9, zitiert nach Warnke 2009: 114). Sprachliche Zeichen sind dabei „sicher die wichtigsten allgemein genutzten Objektivierungsformen von Wissen“ (Köller 2004: 249), um sich über eine Vielzahl unterschiedlicher

60 Damit ist nicht gesagt, dass diese kollektive Zweckorientierung immer faktisch gegeben ist, sondern nur, dass sie zum Konzept `Wissen dazugehört und dadurch Einfluss auf die Konstituierung von Inhalten nimmt, indem beispielsweise eine allgemeine (man geht davon aus ...) und nicht persönliche (ich gehe davon aus ...) Sprecherperspektive gewählt wird. Auch in realistischen Modellen, in denen die ungetrübte Sinneswahrnehmung wahrheits- oder auch wissensgarantierend sein soll, basiert diese Gültigkeitsbedingung auf der Annahme, dass die Sinneswahrnehmung bei denselben Kontextbedingungen bei der Mehrheit der Menschen zu gleichen Ergebnissen führt. 
Gegenstände intersubjektiv auszutauschen, da sprachliche Symbole durch Konvention „intersubjektiv stabile Bezugspunkte für die kommunikative Koorientierung der Individuen und die Selbst- und Fremdzuschreibungen von Intentionen“ bilden (Feilke 1996: 63), aber dennoch flexibel sind im Hinblick auf individuelle und situative Kommunikationsbedürfnisse. Bei unterschiedlichen Interessen kommt es zu agonalen semantischen Kämpfen (vgl. Felder 2006b; 2013; 2015) ${ }^{61}$, aber auch zu wert- oder zweckrationalen Allianzen. Neben der Überzeugung, dass Wissen insbesondere „in massenmedial operierenden knowledgeable societies“ immer machtgebunden und umkämpft ist (Warnke 2009: 113f.) und sich in verschiedenen Sachverhaltsperspektivierungen in Texten und Diskursen manifestiert, wird in dieser Arbeit die Auffassung vertreten, dass es in jeder Gesellschaft oder Gruppe auch ein Bedürfnis nach Koorientierung (vgl. Feilke 1996: 35) und damit stabilen, gemeinsamen Wissensinhalten und einer Verständigung über diese gibt. Das bedeutet, dass es neben den agonalen (sprachlichen) Praktiken erstens auch „sym-pathische“62, konsensuelle, unifizierende (sprachliche) Praktiken geben muss (vgl. zum Konzept der >Unifizierungく Kap. 6.2.1 dieser Arbeit), die kommunikative Mittel der „,Mit-Erfahrung““ (Feilke 1996: 34) bzw. „geteilte Akzeptanz von Erkenntnis“ (Warnke 2009: 113) konstituieren können und zweitens, dass es schon aus Gründen der gegenseitigen Versteh- und Argumentierbarkeit eine Art sprachliches und fach- bzw. alltagesweltliches Basiswissen ${ }^{63}$ geben

61 Felder definiert „semantische Kämpfe“ und „,agonale Zentren“ wie folgt: „Unter ,semantischem Kampf ' wird hier - zunächst allgemein formuliert - der Versuch verstanden, in einer Wissensdomäne bestimmte sprachliche Formen als Ausdruck spezifischer, interessensgeleiteter und handlungsleitender Denkmuster durchzusetzen“ (Felder 2006b: 14). „Unter agonalen Zentren verstehe ich einen sich in Sprachspielen manifestierenden Wettkampf um strittige Akzeptanz von Ereignisdeutungen, Handlungsoptionen, Geltungsansprüchen, Orientierungswissen und Werten in Gesellschaften“ (Felder 2015: 108, vgl. auch Warnke 2009; Spitzmüller/Warnke 2011: 43f.). In Kapitel 7.3 werden Definierenspraktiken im Spannungsfeld von >Agonalität ‘ und >Unifizierung، im Rahmen des Burnout-Diskurses einander gegenübergestellt.

62 Helmuth Feilke (1996) bezieht sich in seiner Monografie zur „Sprache als soziale Gestalt“ in Kapitel 2 „Zur pragmatischen Gestalt der Sprache“ auf den Begriff der „Sympathie“ bzw. auf „die sprachtheoretische Interpretation des Begriffs der ıSympathie““ nach Philipp Wegener ([1885] 1991). Nach dieser werden „Mittel der Kommunikation von den Sprachteilhabern erst in der Kommunikation ,entdeckt (ebd. [= Wegener 1885/1991, T.S.], S. 71), und zwar, weil ein Verhalten erst hier eine Reaktion, ein Verstehen auslösen kann, das dieses Verhalten bewertet. Diese Bewertung geht in die künftige Verhaltensbewertung ein. Ohne diese Rückkopplungsinformation und ihre kontinuierliche Einbeziehung ist zwar Verhalten, aber kein soziales Handeln möglich. Dieses setzt vielmehr die Partizipation an Situationen der Verständigung voraus, aus denen die sym-pathisch, das heißt durch `Mit-Erfahrung` bestimmten Mittel gewonnen werden können“ (Feilke 1996: 31f.).

63 Verschiedene hermeneutische und (diskurs-)linguistische Publikationen haben sich mit diesem vorauszusetzenden, verstehens- und kommunikationsrelevanten Wissen befasst, so zum Beispiel Dietrich Busse, der den „umfassenden epistemisch-kognitiven Hintergrund, der 
muss, das nicht bewusst sein muss, aber zwischen den Zeilen ${ }^{64}$ bzw. Worten durchscheinen kann und auf dessen Grundlage die Perspektivierungs- und Argumentationsspiele stattfinden können.

Auch Foucault unterstreicht die Rolle von (impliziten) Formationsregeln bzw. diskursiven und nicht-diskursiven Praktiken, „die systematisch die Gegenstände bilden, von denen sie sprechen“ (Foucault [1969] 1981: 74) und die das Auftreten von Folge-Aussagen im Diskurs mit beeinflussen. ${ }^{65}$ In der Diskursanalyse geht es nach Foucault folglich darum, die „diskursive Formation“ (Foucault [1969] 1981: 67) aufzudecken, indem man die „Gesamtheit von [...] Beziehungen“ (Foucault [1969] 1981: 67) zwischen den sich an der Diskursoberfläche manifestierenden Zeichen in ihrer Regelmäßigkeit und Wiederkehr (Foucault [1969] 1981: 44) analysiert. Um die Qualitäten dieser Zeichenbeziehungen differenziert deuten zu können, wird dieser Arbeit das Zeichenmodell von Ch. S. Peirce zugrunde gelegt, welches im folgenden Kapitel anhand eines Beispiels aus dem Burnout-Diskurs erläutert wird.

\subsubsection{Das Zeichenmodell nach Charles Sanders Peirce}

Der semiotische Denkansatz von Charles Sanders Peirce fußt auf dem Moment der Rückbezüglichkeit von Zeichen auf Zeichen im Prozess der Sinnkonstitution und der Grundüberzeugung, dass jeder Gedanke ein Zeichen ist und ,Zeichen [...] immer auf Gedanken (`thoughts $\triangleleft$ ) bezogen“ bleiben (vgl. Nagl 1992: 22 und 23;

das Verstehen einzelner sprachlicher Zeichen(ketten) oder Kommunikationsakte überhaupt erst möglich macht“ unter dem Begriff ,Kontext“ fasst (Busse 2007: 82). Bern U. Biere reflektiert im Überblick die „Vorstellung der Wissensbasiertheit des Verstehens“ in der hermeneutischen Tradition (Biere 2007: 19f.). Feilke hat den Begriff des Common Sense, der uns ein „fraglos sicheres und unserer Umwelt angepaßtes Handeln“ erlaubt, auf die Sprache angewandt (Feilke 1993: 8). Sprachlicher Common sense ergibt sich dadurch, dass in kommunikativen Beiträgen Ausdrucksarrangements entstehen, die ,die Kontingenz des grammatisch und semantisch möglichen Zeichenausdrucks durch eine Ausdruckstypik“ (Feilke 1993: 16) ersetzen und dadurch zu „Ressourcen des Vorverständigtseins“ (ebd.: 15) werden.

64 Vgl. zur Auffindung dieses Wissens u. a. die Methoden, die Peter von Polenz in seiner Satzsemantik als „Grundbegriffe des Zwischen-den-Zeilen-Lesens“ beschreibt (von Polenz ${ }^{32008)}$ und vgl. auch die Ausführungen von Marcus Müller zum Begriff der `Spur und des `Spurenlesens (Müller 2015: $47 \mathrm{ff}$. und 53ff., siehe auch Scherner 1994 und Kap. 4.1.2 dieser Arbeit).

65 Dieser Zusammenhang von Praktiken und Wissen bei Foucault wird in Kap. 4.1.3 noch ausführlich dargestellt und mit dem Analysekonzept der Praktik/Praxis, das dieser Arbeit zugrunde liegt, verbunden werden. 
vgl. CP 5.253 und 5.28766). Das heißt, dass ein unmittelbar erfahrener Schmerz, sobald man sich auf ihn bezieht, zeichenvermittelt ist (vgl. Nagl 1992: 22) und dass wir unsere Repräsentationen von Dingen nie „direkt mit den Dingen vergleichen können, sondern allenfalls mit anderen Repräsentationen von ihnen“ (Köller 2004: 244). Das heißt nicht, dass jenseits der Zeichen nichts existiert. Denn irgendein potenziell als Schmerz $\mathrm{zu}$ interpretierendes Empfinden motiviert ${ }^{67}$ schließlich den Zeichenbezug. In Peirce Zeichen-Modell beeinflussen sich Zeichenträger (sign), Zeichenobjekt (object) und Zeicheninterpretant (interpretant) wechselseitig, um zu erklären, wie Realität über Zeichen fassbar wird und wie Bedeutung und Sinn im Prozess einer „kontinuierliche(n) Interpretation von Zeichen durch Folgezeichen“ entstehen können (vgl. Köller 2004: 244; Nagl 1992: 32f.). Die triadische Struktur lässt sich in ihren Grundzügen folgendermaßen abbilden (siehe Abbildung 2):

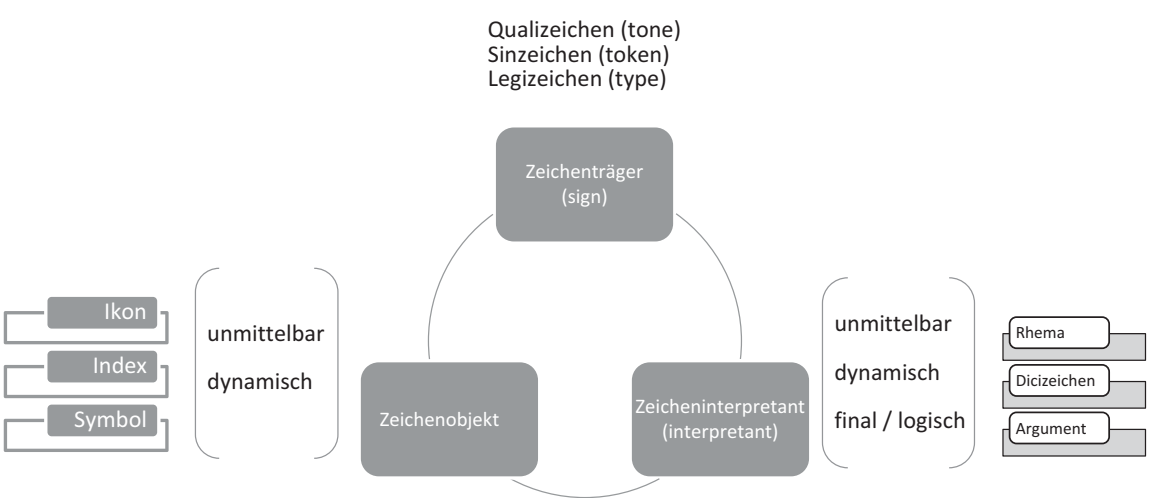

Abb. 2: Das Peirce'sche Zeichenmodell (vgl. Peirce CP 2.228 und Nagl 1992: 43).

Die Abbildung basiert auf „Die Struktur des Peirceschen Zeichenbegriffs“ nach @ Nagl 1992: 43, die Kreisform wurde von der Verfasserin eingefügt, Zahlen wurden nicht übernommen, zu den deutschen Benennungen (Zeichenträger, etc.) vgl. auch Köller 2004: 243.

66 In deutscher Übersetzung in Peirce (1967: 175 und 201).

67 Peirce benutzt das Verb to determine zur Beschreibung der Beziehung zwischen „Objekt und Zeichen einerseits“ sowie „Zeichen und Interpretant“ andererseits (Nagl 1992: 31). Nagl referiert mit Bezug auf die Peirce-Interpretationen von G. Schönrich und M. Frank die Auffassung, dass to determine „nicht in einem engen Sinn verstanden werden dürfe“ (ebd.). Das Verb to determine indiziere „keinen strengen Ursache-Wirkungszusammenhang innerhalb der Zeichenrelata“, sondern sei am besten mit motivieren zu übersetzen: „Das Objekt motiviert - d. h. veranlaßt (ohne rigiden kausalen Zwang) - das Auftreten eines Zeichens, welches selbst wiederum einen ,Interpretanten“ motiviert.“ (Nagl 1992: 31, Fußnote im Zitat entfernt, die Anführungszeichen wurden der Notation dieser Arbeit angepasst). 
Anhand der in der Abbildung dargestellten Größen werden im Folgenden an einem Bild-Text-Beispiel ${ }^{68}$ zum Thema „Burnout“ (siehe Abbildung 3) die für diese Arbeit relevanten Zeichendimensionen durchgesprochen, um im Anschluss daran zu begründen, welche Vorteile das Zeichenmodell von Peirce für die vorliegende Untersuchung bietet und welcher Diskursbegriff sich daran anschließen lässt.

\title{
Burnout Syndrom - Krankheitsbild totaler Erschöpfung
}

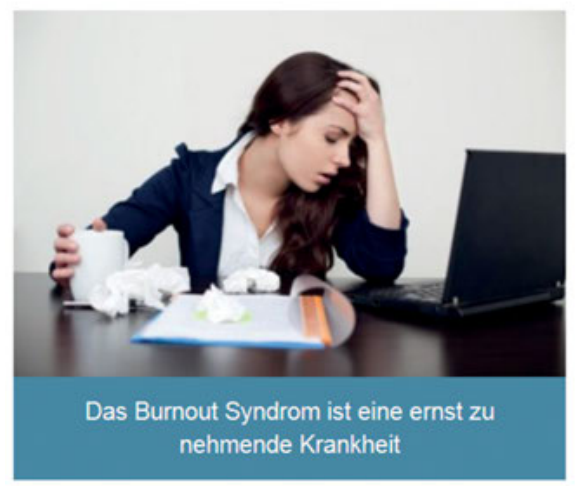

\begin{abstract}
Burnout gilt als Phänomen der heutigen Zeit. Der englische Begriff kann übersetzt werden mit „Ausgebranntsein“. Damit ist ein Zustand völliger Erschöpfung definiert, in dem normale Erholung in gewohnter Manier (z. B. Urlaub) nicht mehr möglich ist. Die Person ist - scheinbar plötzlich zu keiner der Leistungen mehr fähig, die er oder sie bis dahin typischerweise in extrem hohem Maße erbracht hat. Neben der körperlichen Entkräftung findet sich beim Burnout Syndrom die Energielosigkeit auch auf der emotionalen und geistigen Ebene. Vorangegangen ist diesem Zustand eine Vielzahl hochbelastender Stresssituationen.
\end{abstract}

Abb. 3: Bild-Text-Beispiel zum Thema „Burnout“, (C) shutterstock_125936975/baranq, DasBurnoutSyndrom.de

(Quelle: http://www.das-burnout-syndrom.de/, siehe auch Fußnote 68).

Zeichenträger, also das „sign“ im engeren Sinn, sind dieses Bild, die Buchstaben und ihre jeweilige Anordnung, Farbgebung und Typografie. Ein Qualizeichen ist für Peirce dasjenige, was an einem Zeichen sinnlich rezipiert werden kann, d.h. seine Qualitäten. In diesem Beispiel sind dies die verschiedenen Farben- und Formkombinationen des Bilds und der Buchstaben (vgl. Nagl 1992: 52). Mit Sinzeichen ist das singuläre, verwirklichte Vorkommen der jeweiligen Qualizeichen gemeint (vgl. den Begriff der >Token«, CP 2.245). Ein Legizeichen hingegen reicht über die Aktualität seiner Verwendung hinaus (vgl. Nagl 1992: 53).

68 Der folgende Screenshot entstammt der Seite http://www.das-burnout-syndrom.de/ (zuletzt eingesehen am 17.5.2016). Das Bild dieser Internetseite wiederum stammt von der Plattform ,shutterstock“ und wurde für diesen Druck lizenziert (23.9.2021): http://www.shutterstock.com/pic125936975/stock-photo-sick-woman-at-work-with-headache.html?src=gZNNOjfjo7JfO1wXdtqvzg-1-0. 
„Ein Legizeichen ist ein Gesetz, das ein Zeichen ist. Jedes konventionelle Zeichen ist ein Legizeichen. Es ist kein singuläres Zeichen, sondern ein allgemeiner Typ, von dem Übereinkunft darüber besteht, daß er Bedeutung hat“ (CP 2.246). Jeder Buchstabe des Alphabets, jedes Wort einer Sprache ist somit ein Legizeichen.

(Nöth 2000: 65)

Doch auch der dargestellten Szene, Körperhaltung und Mimik der Frau kommt eine gewisse Typik und damit konventionelle Bedeutung zu. Dieser Eindruck wird nicht zuletzt dadurch bekräftigt, dass dieses Bild aus einer Bilddatenbank stammt, also bewusst inszeniert wurde, und mit Ausdrücken wie Stress, erschöpft, Arbeit etc. verschlagwortet ist. Weitere Bilder dieser Datenbank zum Suchwort Burnout zeigen eine sehr ähnliche Bildsprache. ${ }^{69}$ Ein leicht gesenkter, auf eine Hand aufgestützter Kopf wird gemäß unserem Erfahrungswissen in vielen Fällen als Ausdruck der Erschöpfung, Müdigkeit oder als Zeichen für Kopfschmerzen etc. gedeutet. Auf das zerknüllte Papier, die Tasse, Kleidung und Laptop wird gleich im Zusammenhang mit Ikon, Index und Symbol weiter eingegangen werden. Text und Bild dieses Beispiels veranschaulichen die Gesetzmäßigkeit von Zeichen (Legizeichen), die gewährleistet, dass Zeichen von Sprechern/Sprecherinnen und Hörern/Hörerinnen „in ähnlicher Weise gebraucht und verstanden“ werden (vgl. Biere 2007: 13).

Diese Leistung, dass ein Zeichen keine Ähnlichkeit oder Affektiertheit zum Objekt $^{70}$ aufweisen muss, sondern „kraft eines Gesetzes“ (CP 2.249) bzw. einer Regelhaftigkeit des Sprachgebrauchs und Interpretierens, dieses bezeichnen kann, ordnet Peirce den sSymbolen zu. Ikonische und indexikalische Zeichenmodi werden dem symbolischen Zeichenmodus heuristisch gegenübergestellt, da sie durch einen direkteren Bezug zum Realen, durch eine „Dingnähe charakterisiert zu sein" scheinen (vgl. Jäger 2010a: 48f.). Ein Zeichen ist nach Peirce ein Index, „wenn es von dem Objekt, auf das es verweist, ,wirklich beeinflusst ' ist (CP 2.248)“ (Nöth ${ }^{2} 2000: 185$ ). Peirce Beispiele für Indices stammen häufig nicht aus

69 Eine Suche auf http://www.shutterstock.com mit dem Suchausdruck burnout ergibt ähnliche Bilder neben Abbildungen, die die Metaphorik des Ausgebranntseins noch direkter durch verkohlte Streichhölzer oder einen rauchenden Kopf darstellen (Suche auf shutterstock zuletzt am 3.11.2019).

70 Zum Objekt-Begriff und der Unterscheidung in „unmittelbar“ und „dynamisch“ (vgl. CP 4.536; Nöth 22000); Das unmittelbare Objekt ist so, wie es das Zeichen selbst repräsentiert. Das mittelbare, reale ist das Objekt außerhalb des Zeichens (Nöth 22000: 63). Es ist aber „selbst niemals ganz ,zeichenfrei“ da. Denn „es ist immer nur durch Zeichen aufweisbar“ (ebd.). Ähnlich äußert sich Ludwig Jäger im Zusammenhang der Beschreibung der „kulturellen Semiosis“. Er konstatiert, dass das „Vorgängige, das zu Bezeichnende“, erst „,in der Nachträglichkeit der Bezeichnung, gleichsam in einer transkriptiven Umschrift, konstituiert“ wird (Kursivsetzung im Original, Jäger 2010b: 307). 
dem Bereich von Sprache und Kommunikation, wie der häufig angeführte Wetterhahn („A weathercock is an index of the direction of the wind“, CP 2.286), der sich je nach Windrichtung ausrichtet, oder ,organische Symptome als Indices für Krankheiten“ (Nagl 1992: 48; vgl. CP 5.473; 8.335). Doch Peirce bezieht die Indexikalität auch auf Sprachzeichen. Als Exempel für einen sprachlichen Index nennt Peirce den deiktischen Ausdruck here als Antwort auf eine Frage in einem Dialog wie “A says to B, 'There is a fire,' B will ask, 'Where?'” (CP 2.305). Im obigen angeführten Beispiel wird Burnout ebenfalls deiktisch in der heutigen Zeit verortet. Damit die arbiträren Sprachzeichen here bzw. hier oder heutige jedoch als lokale oder temporale Indices auf die konkrete Äußerungssituation verweisen können, müssen sie zunächst nach englischen oder deutschen Sprachgebrauchsregeln, sprich als Symbole interpretiert werden. Für Jäger zeigt sich in solchen Beispielen, dass die

indexikalische Referenz und die symbolische Inferenz [...] im Rahmen dessen, was Peirce
Semiosis, das Spiel der Zeichen, nennt, notwendig aufeinander angewiesen [sind]. Sie
wären als je selbstständige nicht in der Lage, irgendeine Form des Bezugs zur transsemioti-
schen Welt herzustellen: Symbole hätten kein Vermögen, den inferentiellen Horizont ihrer
Weltbeschreibungen zu einer wirklichen Weltberührung hin zu überschreiten und so zwi-
schen einem ,Universum der Realität' und einem ,fiktionalen Universum‘ zu unterscheiden;
und Indices wären nicht in der Lage, die Objekte ihrer Bezugnahme als Referenzobjekte
herauszugreifen.
(Jäger 2010a: 50)

Am obigen Text-Bild-Beleg lässt sich die Verwobenheit der verschiedenen Zeichenmodi (ikonisch, indexikalisch und symbolisch) für die Interpretation weiter demonstrieren: Die Fotografie wirkt einerseits als ikonisches Zeichen, weil sie das Zeichenobjekt (vereinfacht) FRAU AM SchrEIBTISCH qua Ähnlichkeit abbildet. Die Fotografie wirkt andererseits als indexikalisches Zeichen für eine Situation, in der dieses Bild wie Peirce schreibt „point by point to nature“ (CP 2.281) aufgenommen wurde. Diese ikonisch eingefrorene Situation enthält weitere Indices: Die Papierknäuel verweisen auf wahrscheinlich verworfene Notizen, Gestik und Mimik der Frau auf ein Unwohlsein, Kleidung und Laptop verorten die Situation im Arbeitskontext etc. Das interpretative Urteil, dass es sich hier um eine Fotografie und nicht um eine reale Person handelt, basiert wiederum auf einer symbolisch vermittelten Praxis, wie mit solchen Bildern interpretatorisch zu verfahren ist. Überschrift und nebenstehender Text konstituieren den weiteren symbolischen Resonanzraum (vgl. Jäger 2010a: 53f.).

Die bisher beschriebenen Zeichenmodi schließen sich nicht aus. Häufig ergänzen sie sich hinsichtlich der „Wirkung bzw. Motivation“, die ein Zeichen sowie Zeichenkombinationen, die für ein oder mehrere Objekte stehen, in einem Anderen hervorrufen (vgl. Nagl 1992: 53). „Zeichen sind Zeichen für jemanden, 
sie werden rezipiert in Wirkungen, Gefühlen, Handlungen“ und Urteilen (ebd. 40). Diese Wirkung bzw. „das bedeutungshafte Resultat“71 des Zeichens erläutert Peirce durch den Begriff des >Interpretanten`:

A sign [...] addresses somebody, that is, creates in the mind of that person an equivalent sign, or perhaps a more developed sign. That sign which it creates I call the interpretant of the first sign. The sign stands for something, its object. ${ }^{72}$

(CP 2.228)

Dieses Zeichen, das sich an jemanden wendet, kann verschiedener Gestalt sein: beispielsweise ein Schmerz im Arm oder auch ein gelesener Text. Der unmittelbare bzw. „emotional interpretant“ (CP 5.475) ist ein noch nicht reflektiertes Gefühl, das ein Zeichen in einem Menschen auslöst oder auch eine „Interpretationsperspektive, die sich bei der Wahrnehmung eines Zeichenträgers kraft konventionalisierter Assoziationen spontan einstellt“ (Köller 2004: 247). Der dynamische bzw. „energetic interpretant“ ist ein gewohnheitsmäßiges, (inneres) Verhalten oder Handeln, welches auf das Zeichen folgt. Fasst man auf eine heiße Herdplatte, so ist die direkte Reaktion auf den Schmerzreiz, dass man die Hand wegzieht. Ein weiteres Beispiel wäre, dass wir gewohnheitsmäßig auf Unkonzentriertheit oder Leistungsabfall mit einer Pause oder einem Gang an die frische Luft reagieren, also mit Praktiken und Techniken, die normale Erholung in gewohnter Manier versprechen, wie der obige Text in Beispiel 1 nahelegt. Wenn die gewohnten Reaktionen jedoch keine Erleichterung bringen, dann muss das Zeichen vor dem kulturell-sozialen und individuellen Erfahrungs- und Denkhorizont bzw. Wissensrahmen sowie im Hinblick auf Situation und Kontext weiter hinterfragt und gedeutet werden. Das Symptom LeISTUNGSABFALL könnte demnach als 'normal' oder 'nicht normal' oder sogar als 'gefährlich' interpretiert werden. Diese „mit Schlüssen operierende, komplexe Zeichenbewegung“ (Nagl 1992: 41) nennt Peirce den logischen bzw. finalen Interpretanten ${ }^{73}$. Köller deutet diese letzte Kategorie des Interpretanten als

71 "Now the problem of what the 'meaning' of an intellectual concept is can only be solved by the study of the interpretants, or proper significate effects, of signs." (CP 5.474).

72 Übersetzung angelehnt an Nöth (2000: 64) mit kleinen Änderungen: Ein Zeichen adressiert jemanden, d.h., es erzeugt im Geiste dieser Person ein äquivalentes Zeichen oder vielleicht ein weiter entwickeltes Zeichen. Dieses Zeichen, das es erzeugt, nenne ich den Interpretanten des ersten Zeichens.

73 Zur weiteren Differenzierung des logischen bzw. finalen Interpretanten in „Rhema“, „Dicizeichen“ und „Argument“ siehe Peirce CP 8.337; Nagl 53f.; Martin Reisigl fasst diese Modi des Interpretanten folgendermaßen zusammen: „Ein Rhema ist ein einzelner Term oder Begriff, bzw. das, was für sich weder wahr noch falsch sein kann. [...] Ein Dicent, bzw. Dicizeichen (von lat. ,dicere', sagen) ist demgegenüber bereits ein komplexeres Zeichen bzw. ein Zeichenkomplex. Es ist - grob gesprochen - eine behauptete Proposition, die wahr oder falsch sein kann. [...] Ein Argument ist schließlich eine noch komplexere Entfaltung des ,logischen Interpretanten'. Ein 
diejenige Interpretationsperspektive, die im Verstehensprozess der Relation Zeichenträger Zeichenobjekt abschließend zugeordnet wird und die dann den Objektbildungsprozess bzw. Sinnbildungsprozess vorerst einmal beendet.

(Köller 2004: 247)

Vorerst, da dieser Sinnbildungsprozess theoretisch als unabschließbare Wahrheitssuche bei Peirce gedacht wird, die aber dennoch nach dem regulativen Ideal „eine[r] endgültige[n] Meinung“ (vgl. CP 8.148) strebt (vgl. auch Nagl 1992: 42).

Im Modell von Peirce können Zeichen und damit auch zeichenvermitteltes Wissen als „dynamisches Sinnbildungsereignis“ verstanden werden (Köller 2004: 244), denn ihr Sinn wird durch einen interpretativen rückbezüglichen Prozess von Zeichen-Zeichen-Beziehungen konstituiert. Entscheidend für die Interpretation sind neben dem Zeichenobjekt und einzelnen Zeichen andere Zeichen und die mit ihnen verbundenen Sprachgebrauchsregularitäten und kulturell-sozialen Deutungstraditionen. Der Semiotiker nach Peirce ist ein Hermeneutiker, denn „die Bedeutung sprachlicher Zeichen kann [...] nur durch deren Übersetzung in andere Zeichen ermittelt werden" (Nöth 2000: 418). Die Kreisform im obigen Zeichenmodell wurde gewählt, um auf die Verwandtschaft der Peirce'schen Zeichentheorie und die darauf fußenden Schlussmethoden der Abduktion und Induktion mit dem hermeneutischen Zirkel hinzuweisen. So wie beim hermeneutischen Zirkel aus „einzelnen Worten und deren Verbindungen“ das „Ganze eines Werks“ verstanden werden soll und „das volle Verständnis des Einzelnen schon das des Ganzen“ voraussetzt (vgl. Dilthey 1900: 201; vgl. dazu auch Nöth 22000: 419), so gewinnen wir Erkenntnis über eine überraschende Tatsache nach Peirce nur, indem wir über das Auftreten der Tatsache hypothetische Erklärungsregeln bilden und diese dann induktiv an den Daten überprüfen (vgl. CP 5.171).

Die in diesem Kapitel dargelegten semiotischen Modi und Funktionen werden im folgenden Kapitel auf die Begriffe `Diskurs`, ২Diskursanalyse `Hermeneutik bezogen, um das Diskursverständnis und die Basis diskurslinguistischer Analyse, die dieser Arbeit zugrunde liegen, zu verdeutlichen.

\subsection{Zusammenfassung: Anwendung auf das Untersuchungsthema: Linguistisch-hermeneutischer Zugang zum Diskurs über das Phänomen BuRNOUT}

Analog zur Funktionsweise der Semiose wird die diskursive Konstituierung von intersubjektiv geteilten Inhalten und damit von Wissen als regelgeleiteter sozialer, sich in

nach bestimmten Denkregeln aufgebauter Syllogismus oder ein nach bestimmten Regeln konstruiertes Kunstwerk sind dem Peirceschen Verständnis nach ,Argumente““ (Reisigl 2010: 94-95). 
Zeichen vollziehender, rekursiv-zirkulärer Prozess verstanden. In der Anwendung der Peirce’schen Theorie auf den Diskurs wird Diskurs als „Superzeichen““74 (Reisigl 2017: 24) bzw. Zeichenkomplex betrachtet, dessen semiotische Ebenen und Unterebenen (insbesondere die symbolisch-semantischen und -grammatischen sowie indexikalischen Zeichenebenen) in ihrem punktuellen Vorkommen und in der Zusammenschau als Fläche ${ }^{75}$, d.h. in der Gesamtheit ihrer Beziehungen (vgl. Foucault [1969] 1981: 67) gesichtet werden müssen, um den Prozess der Wissenskonstituierung in einem Diskurs zu einem Thema nachzuzeichnen. Auch Spitzmüller/Warnke gehen in ihrer Einführung zur Diskurslinguistik von der semiotischen Organisation des Diskurses aus - dieser besitzt für sie „eine syntaktische, semantische und pragmatische Organisation“ -, um im Anschluss daran die einzelnen Analyseklassen (wortorientierte, propositions- und textorientierte Analyse) als Segmente der intertextuellen Analyse-Ebene vorzustellen (Spitzmüller/Warnke 2011: 138ff.).

Wichtig ist die doppelte Sichtweise auf den Diskurs einerseits als Ereignis und andererseits als „Formationssystem“ (Foucault [1969] 1981: 74), in das sich die Regelmäßigkeit diskursiv-semiotischer Praxis eingeschrieben hat (vgl. Foucault [1969] 1981: 108 und Kap. 3.4.3). Diese zweifache Blickrichtung findet sich auch im Kategorienpaar der kommunikativen und kognitiven Perspektivität nach Köller wieder (Köller 2004; vgl. Kap. 2.1.2). Die kommunikative Perspektivität unterstreicht neben den sich wiederholenden, diskursprägenden Praktiken die Möglichkeit „neue[r] Oberflächen des Auftretens“, die für Foucault Ausgangspunkt für die Transformation von Diskursen darstellen (vgl. Kap. 3.4.3).

Über die Peirce'sche Zeichendimension des Interpretanten wird deutlich, „dass es keine Repräsentation ohne Interpretation“ gibt (Köller 2004: 243) und dass der jeweilige Diskurs- und Denkhorizont entscheidend für die Sinnbzw. Wissenskonstiuierung zu einem Thema ist. Unter >Themen der vorliegenden Arbeit - in Anlehnung an Konerdings Adaption des aus der Gesprächsanalyse stammenden Konzepts von Ochs-Keenan/Schieffelin - „spezifikationsbedürftige Wissensbestände“ verstanden, „für die eine sprachvermittelte Spezifikation" von mindesten einem Akteur, der sich an einen anderen

74 Diesen Ausdruck wählte Reisigl in seinem Vortrag „Diskurssemiotik nach Peirce“ auf der 4. Jahrestagung „Diskurs - semiotisch“ des Netzwerks „Diskurs interdisziplinär“ am 04.12.2014 in Mannheim. Das Programm der Tagung ist einsehbar unter: http://www1.ids-mannheim.de/ fileadmin/lexik/SprachlicherUmbruch/4_Jahrestagung_Netzwerke_2014_12.pdf, Stand Mai 2016. 75 Andreas Gardt entwirft in seinem Aufsatz „Stil und Bedeutung“ das semantische Kategorienpaar der punktuellen und flächigen Bedeutungskonstitution. Dieses wird im Laufe dieses Kapitels noch einmal aufgegriffen und erläutert werden (Gardt 2009: 1202). 
Akteur oder ein Kollektiv textuell wendet, „intendiert bzw. vorgesehen ist“. ${ }^{76}$ Konerding führt dazu weiter aus:

Sprachlich können derartige Themen - in prototypischer Form - in einer Frage explizit Ausdruck erlangen, der sog. ,Question of immediate concern' (Och-Keenan/Schieffelin 1976). Antworten auf derartige implizit oder explizit geäußerte Fragen sind in Form von Texten oder Textteilen (bzw. Gesprächsabschnitten) gegeben.

(Konerding 2007: 110)

Des Weiteren seien, so Konerding, „Texttypen primär deskriptiver und primär argumentativer Natur“ zu unterscheiden: In ersteren findet eine Aufschlüsselung unterspezifizierter Details bestimmter Komponenten statt. Textthemen dieser Art lassen sich ,,in so genannten Ergänzungsfragen bzw. ,w-Fragen““ ausdrücken. In argumentativen Texten hingegen werden „übergeordnete Wissenszusammenhänge gefordert“, die der Argumentation und damit „rezipientenbezogene[n] Akzeptanz“ dienen können. Diese Texte basieren auf „so genannten Entscheidungsfragen (ja/nein-Fragen), für die zusätzlich Begründungen gefordert sind“ (ebd.).

Am Anfang ${ }^{77}$ des Burnout-Diskurses stehen passend zu diesen Erläuterungen zum Begriff des Themas explizit geäußerte Fragen. Herbert Freudenberger stellt im ersten Abschnitt seines im Diskurs häufig als Gründungstext titulierten Beitrags „Staff Burn-out“ die Frage: „What ist burn-out?“ und dieser Frage folgt ein Katalog weiterer Fragen (vorwiegend $w$-Fragen): „What are its signs, what type of personalities are more prone than others to its onslaught? [...].“ (Freudenberger 1974: 159, im QV unter 8.1.5). Freudenberger inszeniert diese Fragen als Reaktion auf ein im Gespräch mit Kollegen über ein in ehrenamtlichen Klinikeinrichtungen neu erlebtes Erschöpfungs-Phänomen (ebd.), das er sich zunächst nicht erklären kann. In Anlehnung an das Zeichenmodell von Peirce könnte man sagen, Freudenberger differenziert mithilfe des Zeichens Burn-out eine „Teilgröße aus dem Kontinuum der physischen und der geistigen Welt“ heraus (Köller 2004: 243), die gleich eines Signals (vgl. das Kap. 3.1. zur med. Semiotik) nach Erklärung ruft, d. h. spezifikationsbedürftiges Wissen darstellt. Damit ist ein Thema konstituiert, das, dadurch dass es von anderen Autorinnen und Autoren textuell aufgegriffen wird, potenziell diskursprägend ist. Denn unter Diskurs wird in dieser Arbeit im Anschluss an Felder ein ,Textund Gesprächsnetz zu einem Thema“ verstanden (Felder 2012: 122), wobei diese Arbeit sich auf schriftliche Texte konzentriert. Nach Busse/Teubert kann

76 Konerding (2007: 110) Bezug nehmend auf Ochs-Keenan/Schieffelin 1976.

77 Die Begründung, aus welchen Gründen der Text von Freudenberger als Beginn des BurnoutDiskurses gelten kann, erfolgt in Kapitel 5.1.2. 
man dieses Textnetz weiter spezifizieren. $\mathrm{Zu}$ einem Diskurs gehören laut ihnen alle Texte, die:

- $\quad$ sich mit einem als Forschungsgegenstand gewählten Gegenstand, Thema, Wissenskomplex oder Konzept befassen, untereinander semantische Beziehungen aufweisen und/oder in einem gemeinsamen Aussage-, Kommunikations-, Funktions- oder Zweckzusammenhang stehen,

- den als Forschungsprogramm vorgegebenen Eingrenzungen in Hinblick auf Zeitraum/ Zeitschnitte, Areal, Gesellschaftsausschnitt, Kommunikationsbereich, Texttypik und andere Parameter genügen,

- $\quad$ und durch explizite oder implizite (text- oder kontextsemantisch erschließbare) Verweisungen aufeinander Bezug nehmen bzw. einen intertextuellen Zusammenhang bilden.

(Busse/Teubert 1994: 14)

Anhand dieser Kriterien wurde ein Korpus zusammengestellt (zur Korpuserstellung siehe Kap. 5), um zu untersuchen, wie sich die Entstehung von Wissen zu einem Phänomen im Bereich von Gesundheit und Krankheit am Beispiel BURNOUT semiotisch-diskursiv vollzieht bzw. an der Sprachoberfläche manifestiert, welche sozialen und kulturellen Rahmenbedingungen in die Wissenskonstituierung mit hineinspielen und welche Perspektiven im Diskurs dominant versprachlicht werden.

Als „besonders relevante Beobachtungsräume“ (Müller 2007: 89) zur Beantwortung dieser Erkenntnisziele dienen in der folgenden Analyse Diskursabschnitte, in denen das Phänomen im weiteren Sinne definiert, d.h. ab-, ein- und umgrenzt wird (vgl. zur weiteren Begründung der Analyseausschnitte insbesondere Kap. 6.2.1 dieser Arbeit). Als Werkzeug der Beschreibung dieser sich in den Definitionsprozessen verdichtenden Wissensproduktion wird das sozial- und kulturtheoretische Konzept der >Praxis` bzw. >Praktiken` für die Diskursanalyse fruchtbar gemacht (Kap. 4.1). Denn dieses eignet sich besonders gut, um den diskursiven Prozess der Wissenskonstituierung im Wechselspiel inner- und außerdiskursiver Elemente, reflexiver und vorreflexiver Verfahrensweisen sowie sozialer, konventioneller und individueller Momente im Bereich von Gesundheit und Krankheit zu beschreiben.

Der Analysezugang erfolgt stets über die Sprachoberfläche. Dabei liegt das Hauptaugenmerk auf den Sprachzeichen(komplexen) bzw. der „vertextete[n] Sprache von Diskursakteuren, die auf der Basis von Textsortenroutinen im Medium Sprache handeln“ (Felder 2012: 120). Denn diese Sprecher/innen versuchen auf der Grundlage von symbolischem Wissen, d. h. ihrer Kenntnis des Sprachsystems und „Sprachgebrauchserfahrungen (Wissen über Verwendungsweisen von Wörtern, Syntagmen usw.)“ (Felder 2012: 117) Rezipientinnen und Rezipienten Erfahrungen und Inhalte zum Phänomen BuRNout zu vermitteln. 
Letztere verstehen und interpretieren diese Zeichen im Ko(n)text, vor ihrem gegenwärtigen Wissenshorizont und ihren eigenen sozial-kommunikativen Sprachgebrauchserfahrungen. Als Analysandin des Diskurses habe ich ebenfalls die Rolle der Rezipientin inne. Mithilfe transparent zu machender Instrumentarien (siehe Kap. 4 und 6.2.1) untersuche ich die sprachliche Diskursoberfläche der als relevant beurteilten definierenden Diskurssequenzen im Hinblick auf Zeichenformen und -kombinationen, die ins Auge springen (vgl. Gardt 2009: 1203). Daraufhin bilde ich mir im Sinne der semiotischen und hermeneutischen Tradition abduktiv ${ }^{78}$ Vorurteile $^{79}$ über die Bedeutung und das Gemeinte bzw. den Sinn ${ }^{80}$ der Texte oder Diskursbestandteile und stelle „Vermutungen über Regularitäten im diskursiven Sprachgebrauch“ (Felder 2012: 126) zum Prozess des Definierens und zur Verortung eines Phänomens zwischen den Polen `Gesundheit ` und `Krankheit ` am Beispiel BuRnouT auf. Diese vermuteten semiotischen Regularitäten gilt es im Hinblick auf ihr semantisches und pragmatisches Potenzial durch empirische Überprüfung zu plausibilisieren: Gibt

78 Felder stellt seinem Untersuchungsansatz der pragma-semiotischen Textarbeit die Peirce'sche Annahme ,über die natürliche Affinität (CP 2.776) des menschlichen Geistes zu den Gesetzen oder Regelmäßigkeiten seiner Umwelt“ und das logische Schlussverfahren der Abduktion voran. „Bei der Abduktion schließt der Analytiker nach Peirce von einem erklärungsbedürftigen Resultat auf eine bisher unbekannte und nur probeweise angenommene (hypothetische) Regel, um den vorliegenden Fall zu klären (Abduktion als Imagination der möglichen Gesetzmäßigkeit)“ (Felder 2012: 126; vgl. zur Abduktion nach Peirce auch Nöth 22000: 67). Zudem verbindet Felder in seinem Untersuchungsmodell der ,pragmasemiotischen Textarbeit“ systemsemiotische bzw. -semantische mit pragma-semantischen Ansätzen (Felder 2012).

79 Andreas Gardt erläutert den Begriff des „Vorurteils“ in Anlehnung an Hans-Georg Gadamer für das linguistische Interpretieren als gängige Praktik, dass Leser/innen „noch vor Lektüre“ Vorurteile „an den Text“ herantragen (Gardt 2007: 263). Das Vorurteil ist nach Gadamer „ein Urteil, das vor der endgültigen Prüfung aller sachlich bestimmenden Elemente gefällt" wird, es heißt also „durchaus nicht notwendig falsches Urteil“ (Gadamer 1960: 275; vgl. die Ausführungen dazu bei Gardt 2007: 263).

80 Von Polenz bestimmt „Bedeutetes und Gemeintes“ wie folgt: „Bei bedeuten haben wir es mit einer EIGENSCHAFT abstrakter Dinge (Wörter, Sätze, Zeichen) zu tun, genauer: einer Beziehung zwischen einem Zeichen und einem Inhalt, bei meinen dagegen mit einer kognitiven bzw. kommunikativen HANDLUNG von Sprechern/Verfassern (AGENS)“ (Polenz von ${ }^{3} 2008: 298$ ). Gardt unterscheidet im Rahmen seiner Ausführungen zu „Methoden des linguistischen Interpretierens zwischen „Bedeutung“ und „Sinn“ von Texten: „Gemeint ist die Tatsache, dass Texte niemals nur eine Bedeutung haben, sondern immer auch einen bestimmten Sinn, und das ist etwas Anderes. Während die Bedeutung aus der Kenntnis des Sprachsystems erschließbar ist, kann der Sinn nur aus dem aktuellen Kontext bestimmt werden, der auch Faktoren einschließt wie den Individualstil des Autors, die Tradition von Textsorte bzw. -gattung, die Kenntnis des kulturellen Umfeldes eines Textes etc.“ (Gardt 2007: 276). 
es weitere Zeichenvorkommnisse, welche die Regularität der beobachteten sprachlichen Einheiten bestätigen, bekräftigen oder korrigieren? Wie ist eine entdeckte sprachsemiotische Regularität innerhalb des Diskurses zu deuten als Zeichen für eine langue-Bedeutung, als zentrale und typische Verwendung, Kontextualisierung und Funktion einer Spracheinheit im intersubjektiven Kommunikations- und Verständigungsprozess, als dominante Objektivierung bzw. Perspektive auf einen Gegenstand/Sachverhalt oder als Anzeichen für eine zentrale Denkweise bzw. ein Konzept? Diese Arbeit plädiert mit Felder (2006b; 2012) ${ }^{81}$ für eine Verbindung dieser semantischen Fragestellung zur Untersuchung der Konstituierung und Definierenspraxis von Wissen im Bereich von Gesundheit und Krankheit. Gardt betont zur Frage der Bedeutung in Texten:

Bedeutung in Texten ist ein flächiges Phänomen, das hat sie mit Stil gemein. Sie entsteht was hier immer zu lesen ist im Sinne von: wird vom Leser gebildet - natürlich ganz entscheidend durch die punktuelle Präsenz einzelner Wörter und Aussagen [...]. Aber sie entsteht auch zwischen den Wörtern und Aussagen, durch die Art und Weise ihrer Kombination. [...]. Dabei gilt, dass alles in einem Text potentiell bedeutungstragend ist, seine lexikalischen Elemente, seine grammatischen Formen und alle Gestaltungsmittel, die Elemente und Formen in Beziehung zueinander setzen, einschließlich der Strukturen des Textes als ganzem, d. h. seines Aufbaus, der argumentativen Verknüpfung seiner Segmente. In ihrem Ensemble bilden diese Größen den Stil des Textes.

(Gardt 2009: 1202)

Die Aussagen von Gardt über die Bedeutung in Texten werden hier auf die Bedeutungskonstituierung und damit Wissenskonstituierung $\mathrm{zu}$ einem Thema in Diskursen übertragen. Denn es gilt auch im Diskurs, dass alles potenziell bedeutungsindizierend ist und in Beziehung zueinander gebracht werden kann. Die semiotischen Spuren dieser semantischen Beziehungen sind durch die Diskursanalyse intra- und intertextuell aufzudecken und im Hinblick auf ihre Regularität bzw. Typizität zu überprüfen.

Das Stichwort der semiotischen Spuren ${ }^{82}$ leitet über zum indexikalischen Analysefokus, den Marcus Müller als äußerst erkenntnisstiftend nicht nur für gesprächslinguistische, sondern auch für korpuspragmatische Studien heraus-

81 Felder diskutiert die verschiedenen semantischen Theorieansätze (kognitive Semantik, Referenzsemantik und handlungstheoretische Semantik) zur Untersuchung der Konstituierung fachwissenschaftlicher Sachverhalte in Texten und im Hinblick auf die Analyse von semantischen Kämpfen in Wissensdomänen. Er kommt zu dem Schluss: „Mitunter wird in Abhandlungen der Eindruck erweckt, Gebrauchstheorie und repräsentationistische Theorie seien alternative, sich gegenseitig ausschließende Theorien. Das wird für die vorliegenden Untersuchungen bezweifelt: sie geben nur auf unterschiedliche Fragen Antworten - und die können unter Umständen beide erkenntnisstiftend sein“ (Felder 2006b: 23).

82 Zur genaueren Fassung des Begriffs der Spur, siehe Kap. 4.1.2. 
stellt. Er vertritt die These, „dass Indexikalität die grundlegendste und wichtigste Eigenschaft von sprachlichen Zeichen im Gebrauch ist" und erläutert diese (weite) Auffassung von Indexikalität anhand von Studien aus dem Bereich der interaktionalen Linguistik und Gesprächsforschung (Müller 2015: 51f.). Indexikalität werde im Rahmen der interaktionalen Linguistik als „grundlegendes Prinzip der Verständigung in Gesprächen“ angesehen: ${ }^{83}$

Demnach nehmen die Gesprächspartner durch die Disposition und Art der Hervorbrin-
gung ihrer sprachlichen Ausdrücke wechselseitig Bezug auf die thematische, situative
und soziale Dimension ihrer Interaktion und konstituieren so den Sinn ihrer Konversation.
Diese Anschauung ist in Form der Kontextualisierungstheorie über die interaktionale Lin-
guistik hinaus wirksam geworden.
(ebd.: 52)

Auch in einem schriftlichen Textnetz bzw. Diskurs birgt die Art, wie ein Emittent seine sprachlichen Ausdrücke hervorbringt, indexikalisches Analysepotenzial im Kontext des Diskursraums. Spuren an der Sprachoberfläche können beispielsweise auf den Kontext, in dem ein Thema entfaltet wird, verweisen (z. B. durch die Art des Textaufbaus, der für eine bestimmte fachliche Textsorte steht), sie können Rückschlüsse auf die Einstellung der Emittenten zum Texthema zulassen oder sie verweisen auf andere Stimmen im Diskurs zu einem Thema, z. B. durch negative Formulierungsweisen, die indexikalisch einen Bezug zu fachlich üblichen Einordnungspraktiken herstellen, der an dieser Stelle jedoch zurückgewiesen wird.

In der folgenden Analyse wird es daher auch darum gehen, herauszuarbeiten, welche Spuren (unwillkürlicher) sozio-kultureller Praktiken und Normen der Diskurs aufweist, die auf den Prozess des Definierens und die Wissenskonstituierung und -strukturierung einwirken (vgl. Kap. 6.2). Ziel der Analyse ist es, die sprachlichen Ressourcen und Praktiken zu entdecken, welche die Konstituierung bzw. den Definierensprozess des Phänomens BURNOUT im Diskurs organisieren (vgl. Angermüller 2007: 109). Sprache wird vor diesem Hintergrund mit Müller als „Konglomerat von Spuren sozialer Interaktion“ verstanden (Müller 2015: 47).

In den folgenden Kapiteln werden die bisher dargelegten semiotischhermeneutischen Überlegungen noch differenzierter auf den medizinischpsychologischen Bereich bezogen.

83 Müller bezieht sich dabei insbesondere auf Garfinkel/Sacks 1981 und Mondada 2002 (vgl. Müller 2015: 52). 